\title{
A discrete element/shell finite element coupling for simulating impacts on reinforced concrete structures
}

\author{
Jessica Rousseau* — Philippe Marin** — Laurent Daudeville** \\ Sergueï Potapov***
}

* EDF R\&D, $A M A$

1 av. du Général de Gaulle F-92140 Clamart

jessica.haelewyn@edf.fr

** 3S-R (UJF/INPG/CNRS)

DU BP53, F-38041 Grenoble cedex 9

laurent.daudeville@ujf-grenoble.fr,philippe_marin@yahoo.fr

*** LaMSID (EDF/CNRS)

1 av. du Général de Gaulle F-92140 Clamart

serguei.potapov@edf.fr

\begin{abstract}
The efficiency of the discrete element method for studying the fracture of heterogeneous media has been demonstrated, but it is limited by the size of the computational model. A coupling between the discrete element and the finite element methods is proposed to handle the simulation of impacts on large structures. The structure is split into two subdomains in each of which the method is adapted to the behaviour of the structure under impact. The DEM takes naturally into account the discontinuities and is used to model the media in the impact zone. The remaining structure is modelled by the FEM. We propose an extension of the coupling procedure to connect the Discrete Element model to shell-type Finite Elements. The efficiency of the coupling method is tested and validated.

RÉSUMÉ. L'efficacité de la méthode éléments discrets pour étudier la rupture des matériaux hétérogènes a été démontrée, mais elle reste limitée par les capacités de calcul informatique. Un couplage entre éléments discrets et éléments finis est proposé. La méthode éléments discrets qui prend naturellement en considération les discontinuités est employée pour représenter la zone d'impact. La partie restante de la structure est simulée par éléments finis. Nous proposons une adaptation de la méthode de couplage pour relier le modèle éléments discrets à des éléments finis de type coque. L'efficacité de la méthode de couplage est testée et validée.

KEYWORDS: discrete elements, finite elements, coupling, fast dynamics.

MOTS-CLÉS : éléments discrets, éléments finis, couplage, dynamique rapide.
\end{abstract}

DOI:10.3166/EJCM.19.153-164 (C) 2010 Lavoisier, Paris

EJCM - 19/2010. Giens 2009, pages 153 to 164 


\section{Introduction}

The general framework of this study deals with prediction of reinforced concrete structure response under a severe local dynamic loading such as an impact due to an aircraft, a missile or a near-field explosion. A reliable and efficient design of structures under such a loading needs to take into account both locally discontinuous behaviour (fractures, fragmentation) and the globally elastic response of the structure.

The Discrete Element Method (DEM) (Hentz et al., 2004a) is appropriate for modelling material discontinuities and also very well adapted to dynamic problems. This method does not rely on any assumption regarding where and how a crack or several cracks occur and propagate since the DE model is naturally discontinuous. Nevertheless, computational time is still prohibitive for large Discrete Element Models, and the analysis of industrial-size structures with DEM only seems difficult.

Because in most cases discontinuous phenomena such as fracture and fragmentation are localized right in the vicinity of the impact, it is worth coupling DEM with the standard Finite Element Method (FEM). The latter method is applied onto the part of the structure remaining elastic, allowing a reduction of both modelling and computation times.

In the first part of the paper, the proposed Discrete Element (DE) modelling is presented. The identification process of DE parameters, in both linear and nonlinear range of the constitutive behaviour, is described briefly. Local model parameters are calibrated in order to reproduce macroscopic concrete behaviour.

The coupling DE/FE algorithm has been first developed to couple discrete elements with 3D massive finite elements (Frangin et al., 2006). In the second part of this paper we remind the coupling method and present its adaptation for shell elements. Finally, the efficiency of the coupling algorithm is verified on a series of elastic, static and dynamic tests.

\section{Discrete element model}

\subsection{Constitutive behaviour of concrete modelled by means of discrete elements}

The fundamentals of the used DEM are fully described in (Hentz et al., 2004a). The elements are rigid spheres of different sizes. The centroid positions are randomly generated through use of a special "disordering" technique (based on an algorithm described in Jerier et al., (2009)) that provides a polydisperse assembly with a particular size distribution. The characteristic sizes of elements do not have to be representative of concrete constituents, such as the granular or cement matrix, since the aim is to produce a macroscopic description.

Two types of interactions are defined. Bond-type interactions are defined between two elements not necessarily in contact. During the simulation, additional 
interactions of contact-type can be added. Interactions between two spherical discrete elements are defined by means of normal $K_{n}$ and tangential $K_{s}$ stiffnesses characterizing the elastic behaviour of concrete. "Micro-macro" relations (Hentz et al., 2004a) give the local stiffnesses $\mathrm{Kn}$ and Ks from the Young's modulus and Poisson's ratio. These relations stem from homogenization models (Liao et al., 1997) typically used for regular DE assemblies; they have been modified to take into account both the relative disorder and the interaction surface $S_{\text {int }}$. Equation [1] shows the micro-macro relations applied to determining $\mathrm{K}_{\mathrm{s}}$ and $\mathrm{K}_{\mathrm{n}}$ between two elements $a$ and $b . D_{\text {in it }}^{a, b}$ stands for the initial distance between elements $a$ and $b$, with $\mathrm{R}_{\mathrm{a}}$ and $\mathrm{R}_{\mathrm{b}}$ being the element radii. The $\alpha, \beta$ and $\gamma$ parameters still need to be identified. All details about parameters identification can be found in Rousseau et al. (2008).

$$
\left\{\begin{array}{c}
K_{n}=E \frac{S_{\text {int }}}{D_{\text {init }}^{a, b}} \frac{1+\alpha}{\beta(1+v)+\gamma(1-\alpha v)} \\
K_{s}=K_{n} \frac{1-\alpha v}{1+v}
\end{array}\right.
$$

A modified Mohr-Coulomb criterion [2] associated with softening is used to model the non-linear behaviour of the material. Local parameters, such as local tensile strength $\mathrm{T}$, cohesion $\mathrm{C}_{\mathrm{o}}$ and softening factor $\zeta$ need to be identified from global parameters such as compressive and tensile strengths $\sigma_{\mathrm{c}}$ and $\sigma_{\mathrm{t}}$ and fracture energy $G_{f}$. A classical Coulomb friction constitutive behaviour is used between elements in contact (Figure 1).

$$
\left\{\begin{array}{cl}
f_{1}\left(F_{n}, F_{s}\right)=F_{s}-\tan \left(\Phi_{i}\right) F_{n}-S_{\text {int }} C_{o} & \text { (Sliding criterion) } \\
f_{2}\left(F_{n}, F_{s}\right)=S_{i n t} T-F_{n} & \text { (Tensile fracture criterion) }
\end{array}\right.
$$

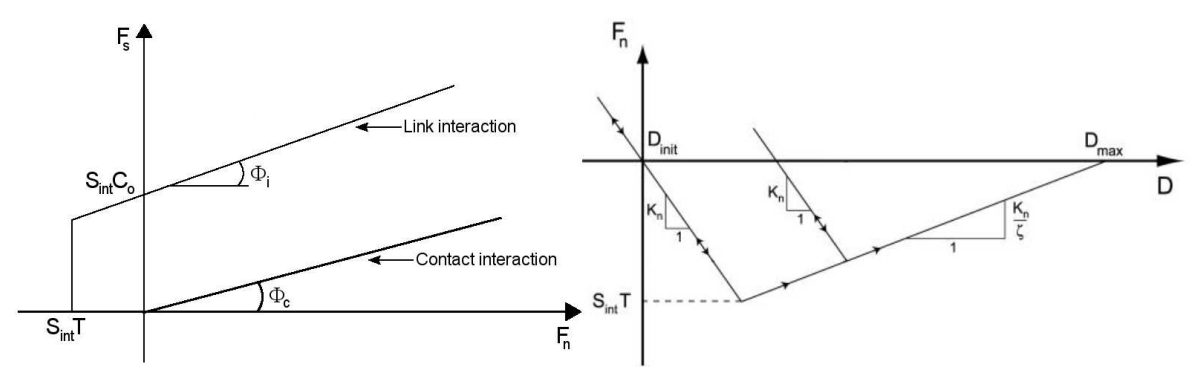

Figure 1. Interaction laws for $D E$

\subsection{Identification of parameters relative to the nonlinear behaviour}

Figure 2 shows the flowchart of the identification procedure allowing to obtain local parameters (local tensile strength $\mathrm{T}$, softening parameter $\zeta$ and cohesion $\mathrm{Co}$ ) through the simulation of uniaxial quasi-static tensile and compressive tests. 


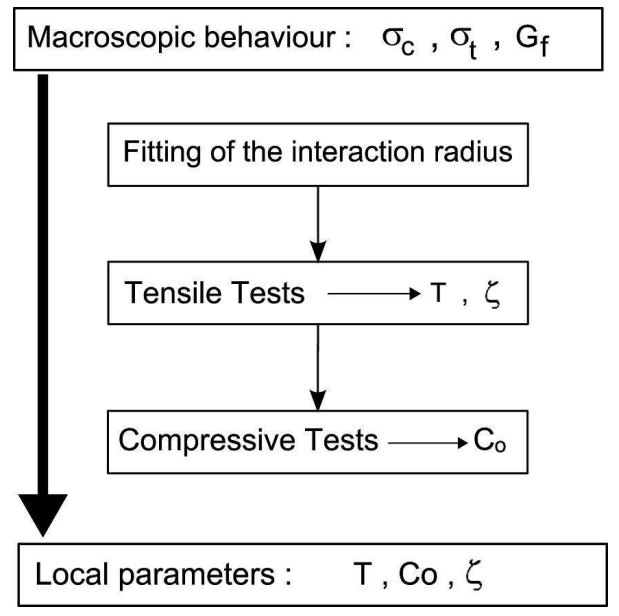

Figure 2. The identification procedure

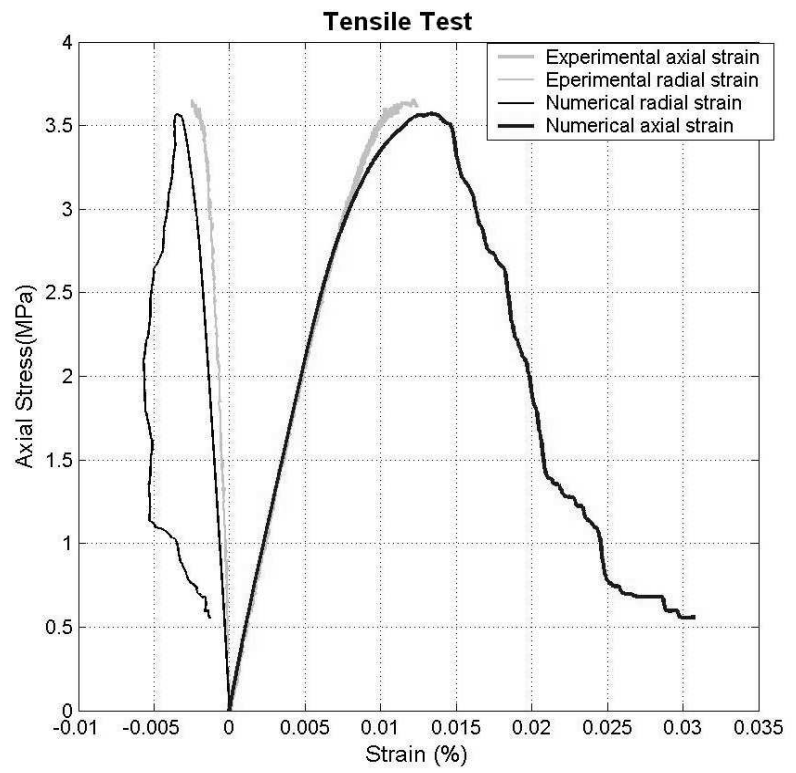

Figure 3. Tensile test

This procedure was applied in order to identify local parameters of a concrete sample extensively studied in the laboratory 3S-R (Gabet et al., 2008). Results of tensile and compressive tests are shown on Figures 3 and 4 . One can see that the use of a local tensile strength $\mathrm{T}$ of $3.3 \mathrm{MPa}$ and softening factor $\zeta$ of 10 provide a good 
approximation of the experimental tensile test (Figure 3). Even though the post-peak part of the experimental curve is not available for updating, the DE model succeeds in reproducing the brittle behaviour typical for the concrete material. The second test involves uniaxial quasi-static compression. Results obtained with the DE model and $\mathrm{Co}=4.4 \mathrm{MPa}$ are in good agreement with the measured data (Figure 4).

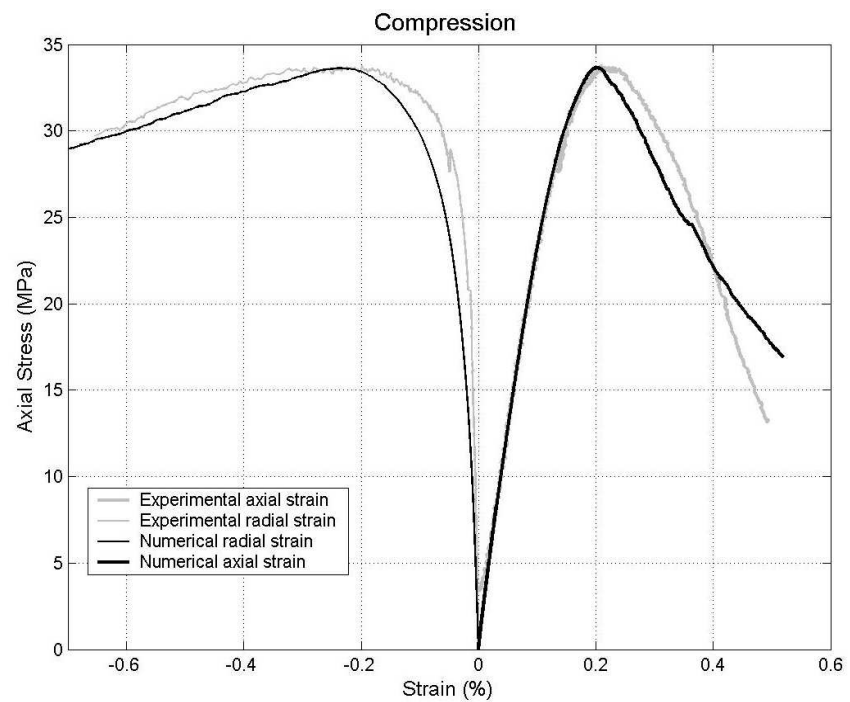

Figure 4. Compressive test

\section{Coupled model}

\subsection{General coupling method}

This section concerns the coupling procedure between continuum and discrete domains. Discrete element method is convenient for modelling discontinuities and fracture but it leads to prohibitive costs of calculation for large structures. To optimize the numerical model, we have chosen to couple discrete elements used in the vicinity of the impact with elastic finite elements used in the rest of the structure.

Many methods have been already developed for multi-scale problems (Xiao and Belytchko, 2004 and Ben Dhia and Rateau, 2005). Xiao and Belytchko, 2004 have introduced the idea of a bridging domain where the Hamiltonian is a linear combination of molecular dynamics and finite elements. Here, we use the bridging domain method to couple DE and FE models (Figure 5). Equation [3] shows that DE and FE Hamiltonians are weighted by a parameter $\eta$ whose value is 1 in the FE domain and 0 in the DE domain.

$$
H=\eta H_{F E}+(1-\eta) H_{D E}
$$




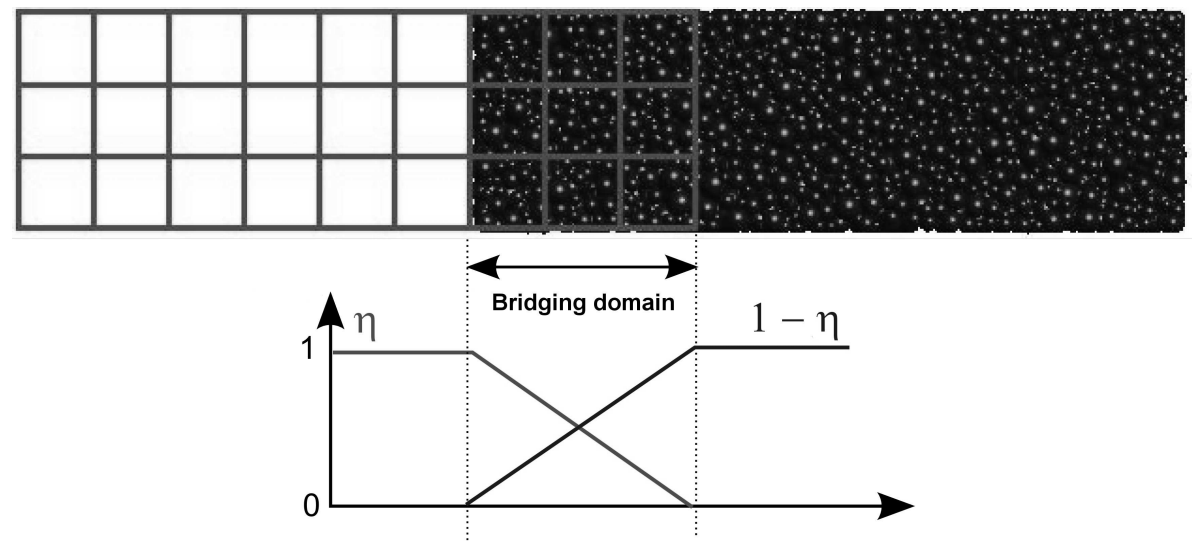

Figure 5. Bridging domain

To guarantee kinematic continuity, the degrees of freedom of elements in the DE and FE domains have to be linked within the interface zone. Several approaches can be considered. Following Xiao and Belytschko (2004), we link the Discrete Element degrees of freedom with Finite Element degrees of freedom using Lagrange multipliers. Equation [4] presents the kinematic conditions in the bridging domain using DE displacements $d_{r}$ and FE displacements $u_{r}$. Details of the method are explained in (Frangin et al., 2006) and (Rousseau et al., 2009).

$$
\vec{d}_{r}=\bar{k} \vec{u}_{r}
$$

The algorithm of DE-FE coupling is implemented in the fast dynamics software Europlexus (Europlexus, User's Manual). In this code, an explicit time integration is used with a formulation based on velocities (Casadei and Halleux, 1995 and Key, 1980). For mixed DE and FE models, the critical time step given by the classical stability condition is imposed by the discrete element model which is the most restrictive due to the small size of discrete elements. The coupling decreases the critical time step as explained in Rousseau et al. (2009).

\subsection{Coupling shell finite elements with discrete elements}

Because the shell elements are more widely used to model large thin structures, we adapt the coupling method presented in the previous section. The difficulty consists in finding the appropriate kinematics constraints between shell nodes displacements and discrete elements displacements. Figure 6 shows an example of possible location of $\mathrm{DE}$ with respect to the shell elements. For the two discrete elements in the middle, the nearest shell element cannot be easily identify: one of them is outside of the two shell elements thickness whereas another one belongs to both shells. 


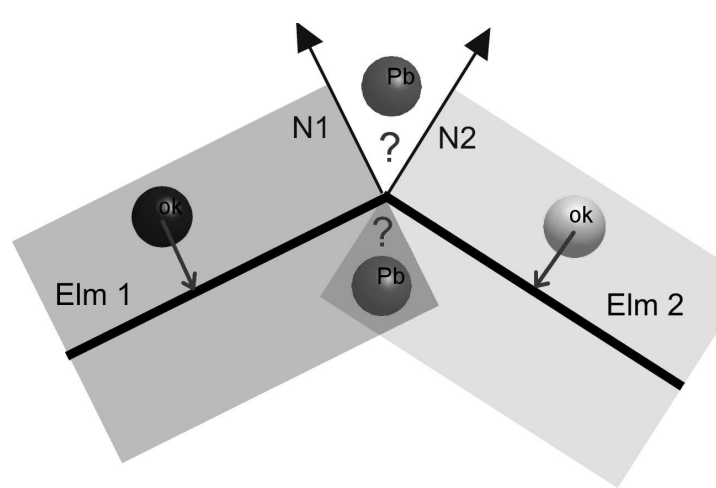

Figure 6. Difficulties in locating DE with respect to shell elements

To solve this problem, we calculate an average normal for all shell nodes. Thus, our main idea is to create fictive nodes and build a fictive $3 \mathrm{D}$ finite element (Figure 7). With those fictive nodes, the previously described coupling method can be applied. Each fictive node is defined using the average normal $\mathrm{n}$ and the real thickness $\mathrm{h}[5]$.

$$
\vec{x}_{\text {fictif }}=\vec{x}_{\text {reel }} \pm \frac{h}{2} \vec{n}
$$

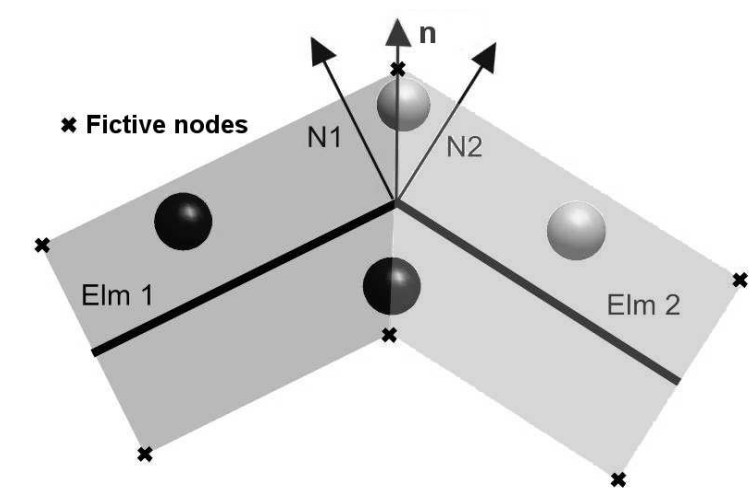

Figure 7. Definition of fictive nodes

The kinematic constraints are formulated using displacements of the fictive nodes and the displacements $\mathrm{u}$ and rotations $\theta$ of real shell nodes are calculated using the standard interpolation equation for shells ((Reissner, 1974) :

$$
\vec{u}_{\text {fictif }}=\vec{u}_{\text {reel }}+z \vec{\theta} \text { with } z= \pm \frac{h}{2}
$$




\section{Validation of the coupling method}

To validate the exposed coupling algorithm, several quasi-static and dynamic tests has been simulated. To reproduce the quasi-static conditions, an artificial damping, proportional to the velocity, is introduced for discrete and finite elements. For all tests considered, three numerical models have been compared: the full FE model, the coupled DE/shell FE model and the full DE model.

We model a concrete bar $(0.85 \mathrm{~m} \times 0.25 \mathrm{~m} \times 0.25 \mathrm{~m})$ with FE shells and DE using three FE layers in bridging domain (Figure 8). One end of the sample is blocked whereas a displacement is applied on the other end.
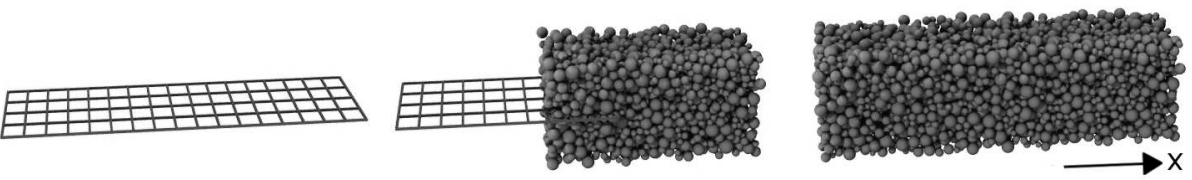

Figure 8. FE, coupled and DE models used in validation tests

First, on Figures 9 and 10, the final deformation shapes of the beam predicted by the three numerical models are compared with the correspondent theoretical solutions. A good agreement of results validates the coupling algorithm in elasticity for both static tension and bending.

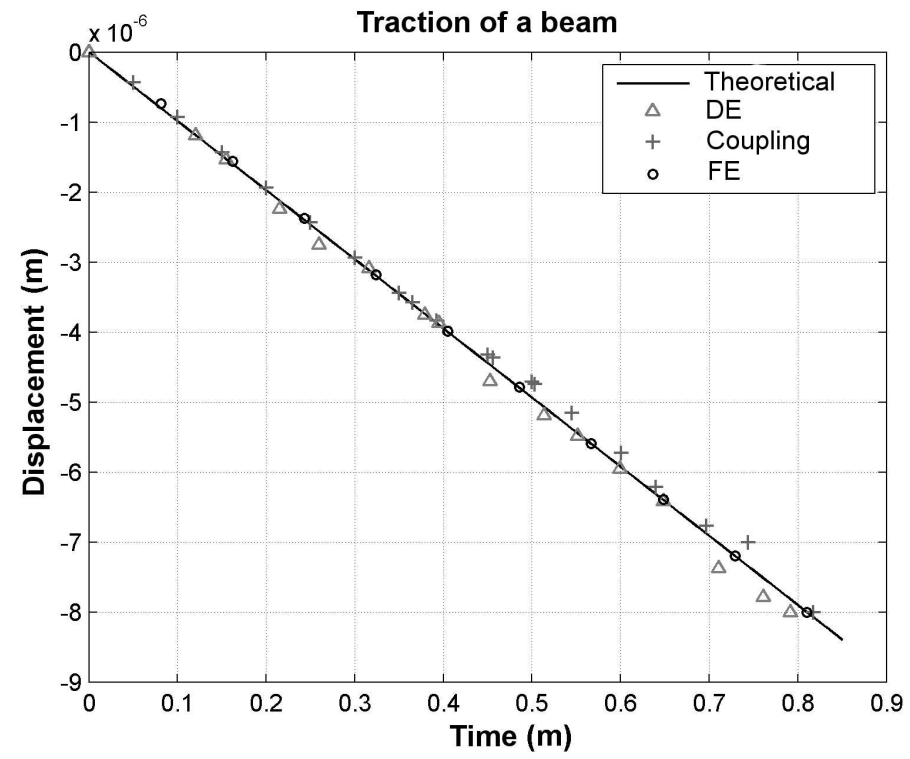

Figure 9. Comparison of results in quasi-static tensile tests 


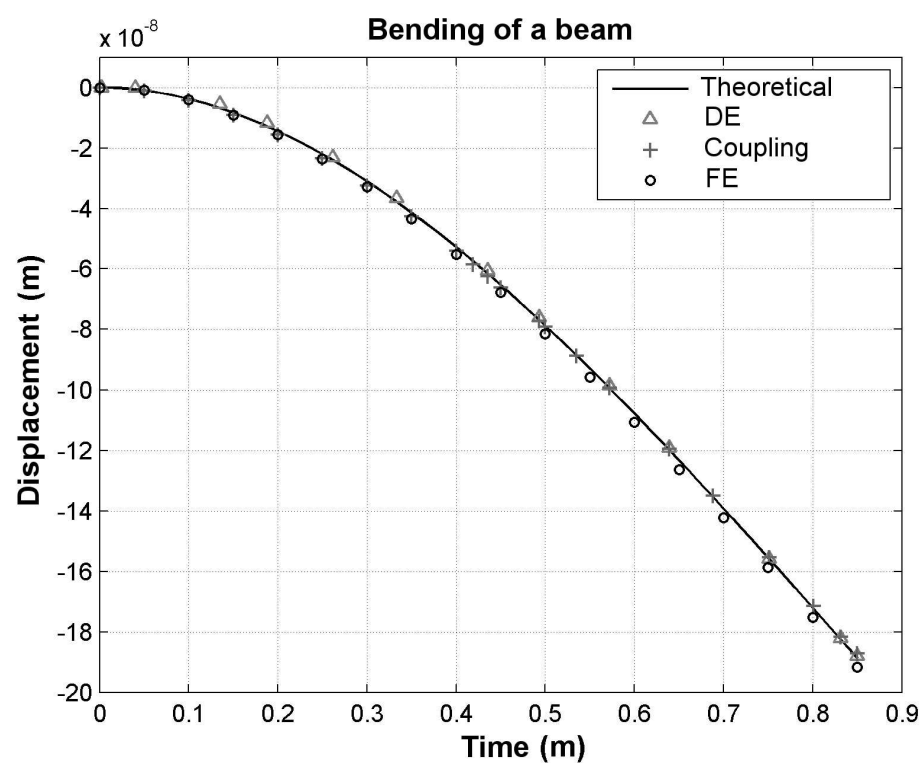

Figure 10. Comparison of results from a bending test

In dynamics, the objective is to evaluate the errors made using the coupling method compared with the finest model i.e. the discrete element model. The displacements are compared for the three models in two points of the beam (Figure 11) situated in the DE zone at $x=0.75 \mathrm{~m}$, and in the bridging domain at $\mathrm{x}=0.4351 \mathrm{~m}$ (DE and coupled models) or $0.45 \mathrm{~m}$ (FE model).

The three models present very similar answers (Figure 12). The difference on the first frequency of oscillation is of $4 \%$ approximately between the coupled model and the DE model and about $1 \%$ between the coupled model and the FE model. The difference in amplitude is about $6 \%$ between the coupled model and the DE model and about $7 \%$ between the coupled model and the FE model. Displacements of the coupled model are between those of the FE and the DE models.

Similarly, in bending, displacements are compared for the three models (Figure 13). Here, the differences on the first frequency of oscillation are about $1 \%$ between the coupled model and the DE model and approximately $3 \%$ between the coupled model and the FE model. The amplitude difference is about $3 \%$ for $x=0.75 \mathrm{~m}$ and $8 \%$ for $\mathrm{x}=0.4351 \mathrm{~m}$ between the coupled model and the DE model. It is approximately $2 \%$ for $x=0.75 \mathrm{~m}$ and $8 \%$ for $x=0.4351 \mathrm{~m}$ respectively for the coupled model and the FE one. It is observed that displacements of the coupled model are always situated between DE and FE model displacements. 


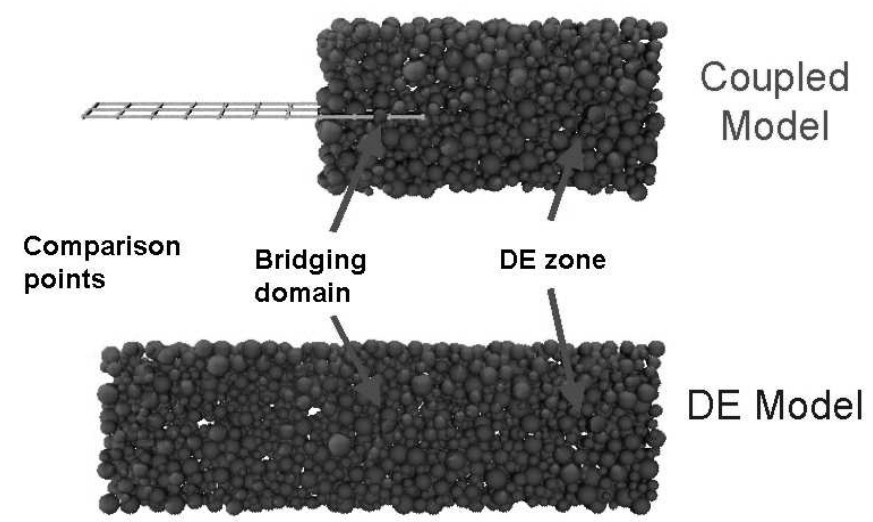

Figure 11. Location of the comparison points for the dynamic tests
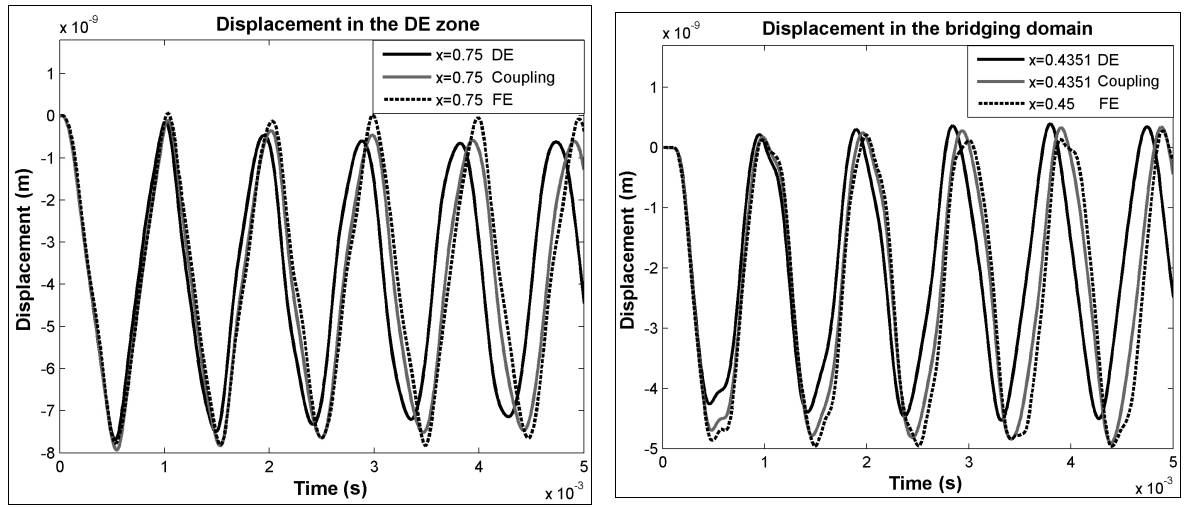

Figure 12. Tension test: axial displacements in the DE zone and in the bridging domain
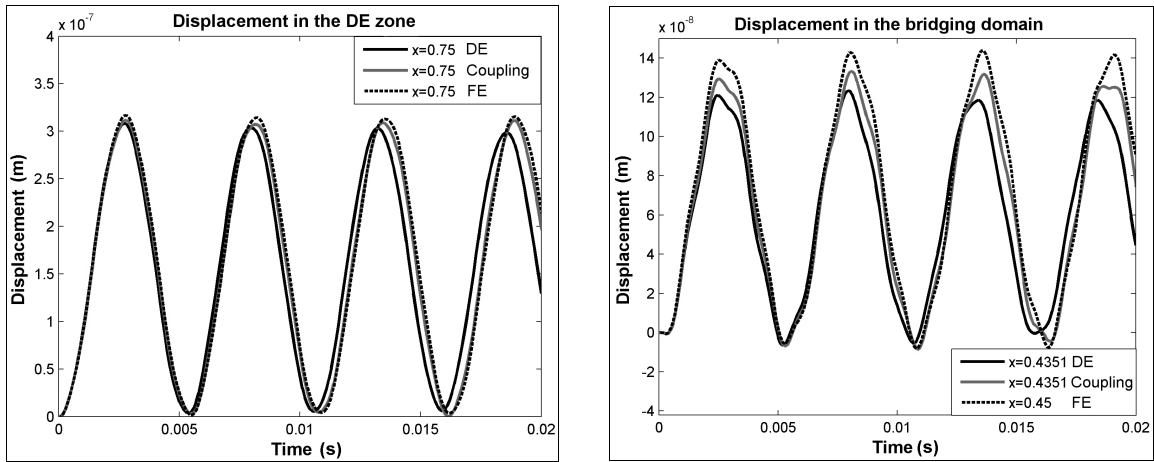

Figure 13. Bending test: vertical displacements in the DE zone and in the bridging domain 
Globally, the elastic responses of coupled DE-FE model in the considered tests are quite close to the reference solutions which proves the efficiency of the coupling method. The presented quasi-static and dynamic tests validate the coupling algorithm in linear elasticity. Next step consists in applying the DE/FE coupling to deal with nonlinear problems.

\section{Conclusion}

The proposed FE-DE coupling algorithm based on a robust identification procedure and the use of an efficient coupling method opens the way to simulate large reinforced concrete structures under impact. It was up to now impossible to simulate such problems by means of discrete elements only. The computational performances can still be improved by parallelizing the discrete element model and by using a multi-step time integration.

\section{References}

Ben Dhia H. and Rateau G., "The Arlequin method as a flexible engineering design tool", Int. J. Numer. Meth. Engng, 62, 2005, 1442-1462.

Casadei F. and Halleux J.P., "An algorithm for permanent fluid-structure interaction in explicit transient dynamics”, Comput. Methods Appl. Mech. Engrg., 128, 1995, 231-289.

Europlexus User's manual. http://europlexus.jrc.eu.europa.eu.

Frangin E., Marin P. and Daudeville L., "On the use of combined finite/discrete element method for impacted concrete structures." J. Phys. IV, 134, 2006, 461-466.

Gabet T., Malecot Y. and Daudeville L., "Triaxial behaviour of concrete under high stresses: Influence of the loading path on compaction and limit states", Cement Concr. Res., vol. $38, n^{\circ} 3,2008,403-412$.

Hentz S., Daudeville L. and Donze F. V., "Identification and Validation of a Discrete Element Model for Concrete", J. Eng. Mech., 130, 2004a, 709-719.

Hentz S., Daudeville L. and Donzé F-V., "Discrete Element Modelling of Concrete Submitted To Dynamic Loading at High Strain Rate”, Comput. Struct., 82, 2004b, 2509-2524.

Jerier J.-F., Imbault D., Donze F.-V. and Doremus P., "A geometric algorithm based on tetrahedral meshes to generate a dense polydisperse sphere packing", Granular Matter, vol. $11, \mathrm{n}^{\circ} 1,2009,43-52$.

Key S.W., Transient response by time integration: review of implicit and explicit operators, J. Donea, ed., Advanced Structural Dynamics (Applied Science, London) 1980, p. 71-95.

Liao, C.-L., Chang, T.-P. and Young, D.-H, "Stress-strain relationship for granular materials based on the hypothesis of best fit", Int. J. Solids Struct., vol. 34, n 31-32, 1997, 40874100 . 
164 EJCM - 19/2010. Giens 2009

Reissner E., Linear and nonlinear theory of shells. Thin Shell Structures, Fung and Sechler Eds., Prentice Hall, 1974, 29-44.

Rousseau J., Frangin E., Marin E. and Daudeville L., "Damage prediction in the vicinity of an impact on a concrete structure: a combined FEM/DEM approach", Computers and Concrete, vol. 5, $\mathrm{n}^{\circ}$ 4, 2008, 343-358.

Rousseau J., Frangin E., Marin P. and Daudeville L., "Multidomain finite and discrete elements method for impact analysis of a concrete structure", Engineering Structures, vol. $31, \mathrm{n}^{\circ} 11,2009,2735-2743$.

Xiao S. and Belytschko T., "A bridging domain method for coupling continua with molecular dynamics”, Comput. Meth. Appl. Mech. Eng., 193, 2004, 1645-1669. 